\title{
Molecular Imaging of Cartilage
}

\begin{abstract}
A

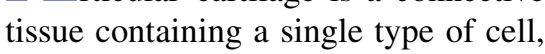
chondrocytes, which synthesize a dense extracellular matrix composed mainly of collagens, hyaluronic acid, and proteoglycans. Proteoglycans represent a class of heavily glycosylated glycoproteins consisting of a core protein with one or more covalently attached chondroitin sulfate chains. These chains are sulfated long linear carbohydrate
\end{abstract}

\section{See page 1541}

polymers (glycosaminoglycans) that are negatively charged because of the presence of sulfate and uronic acid groups. Chondroitin sulfate is an important structural component of cartilage that plays a major role in its in resistance to compression and its elastic properties.

Cartilage proteoglycan targeting by Clermont-Ferrand's team began in an accidental manner. Although studying the biodistribution of the ${ }^{14} \mathrm{C}$-labeled antipoisoning acetylcholinesterase reactivator pyridoxine in rats poisoned by organophosphates (1), the team noticed that after in vivo administration, pyridoxine was concentrated in cartilage and intervertebral disks on autoradiographed whole-body sections of rats. They studied its incorporation into cultured chondrocytes and fibroblasts, as well as binding to macromolecules synthesized by these cells (2), and showed that it was poorly

Received Feb. 11, 2009; revision accepted Feb. 27, 2009.

For correspondence or reprints contact: Dominique Le Guludec, Service de Médecine Nucléaire, Hôpital Bichat, 46 rue Henri Huchard, 75018 Paris, France.

E-mail: dominique.leguludec@bch.aphp.fr

COPYRIGHT (c) 2009 by the Society of Nuclear

Medicine, Inc.

DOI: 10.2967/jnumed.108.057810 concentrated in cells but firmly bound to proteoglycans by ionic interactions.

The binding of amines and several other cations to cartilage was reported as early as $1975(3,4)$ as an ionic-type binding to the anionic carboxyl and ester sulfate groups of chondroitin sulfate, related to electrostatic interaction. Thus, the binding of pyridoxine to proteoglycans of cartilage was attributed to its cationic quaternary ammonium function.

In further investigations, the same team went on developing cartilagetargeting compounds for therapeutic or imaging purposes by linking a quaternary ammonium function to nonsteroidal antiinflammatory drug oxicams (5) and to several polyazamacrocycles that could be labeled with tritium or ${ }^{99 \mathrm{~m}} \mathrm{Tc}$ (6). The team retrieved a high concentration of these compounds from joint cartilage after in vivo administration to rats, particularly for $N$-[triethylammonium]-3-propyl-[15]ane-N5 radiolabeled with ${ }^{99 \mathrm{~m}} \mathrm{Tc}$ ( $\left.{ }^{99 \mathrm{~m}} \mathrm{Tc}-\mathrm{NTP} 15-5\right)$, which demonstrated a ratio of 10 between cartilage and surrounding tissues and high in vivo stability. Studies on chondrocyte cultures confirmed that ${ }^{99 \mathrm{~m}} \mathrm{Tc}$ NTP 15-5 was able to bind to the acidic function of proteoglycans. Therefore, the team went on with a first in vivo imaging study in rabbits with papainand zymosan-induced arthrosis (7) and found a good correlation between tracer uptake and severity of cartilage disease on histology and a significant decrease in uptake before the onset of radiologic abnormalities in the early stage of disease.

\section{ARTHROSIS}

These latter results are of particular interest because arthrosis is the most frequent degenerative cartilage disease, characterized by a progressive and irreversible degradation of matrix components in which one of the earliest molecular events is the degradation of proteoglycans by glycosyltransferases. Because of the hydrophilic properties of its components, a loss of elasticity is induced, with subsequent lesions of collagen fibers and a vicious circle that worsens the lesions. Clinical symptoms appear at a rather late stage of the lesions. Radiologic assessment is not sufficient for assessing the joint cartilage, nor are arthrography and CT. Standardized MRI pulse sequences provide an accurate, reproducible assessment of cartilage morphology, but not until the disease is advanced (8). Detection of metabolites of proteoglycans in the blood or urine is feasible, but sensitivity and specificity are poor.

High-frequency ultrasound was proposed to monitor trypsin-induced progressive cartilage degeneration in cartilage-bone specimens in vitro and provided useful information about the progressive proteoglycan depletion from the early stages of tissue degradation (9). But it cannot be proposed for clinical evaluation.

Recently, some attempts were made to evaluate the spatial distribution and evolution of glycosaminoglycans with delayed gadolinium-enhanced MRI in normal cartilage and repair tissue and with 3-T MRI in matrix-associated autologous chondrocyte transplants of the knee. T1 mapping with $3 \mathrm{~T}$ showed the zonal structure of normal hyaline cartilage, highly reduced zonal variations in repair tissue, and a tendency toward an increase in global and zonal glycosaminoglycan content 1 y after transplantation (10). However, this method represents an indirect evaluation of proteoglycan content.

Therefore, imaging with a labeled specific ligand allowing quantification of proteoglycan loss would be useful for the early detection of arthrosis, longitudinal follow-up of treated patients, and evaluation of new therapies. 
To this end, proteoglycans have been shown to have a major role by way of their ability to retain collagen during cartilage matrix production and integrative repair processes (11). The major challenge will be overcoming the limited sensitivity of scintigraphy in evaluating small changes in proteoglycan content.

\section{CHONDROSARCOMA}

Chondrosarcoma is the most common primary sarcoma of bone in adults, characteristically producing coalescent cartilage lobules of various sizes (12). The most common sites are the pelvic bones, the femur, and the humerus. Other sites are the trunk, the skull, and the facial bones. Conventional radiology allows the depiction and localization of the lesion and identifies its cartilaginous nature and its aggressiveness. CT has a diagnostic role because it shows the bone destruction, the small calcifications, and the intra- and extraosseous extent.

MRI shows typical forms of the lesions as lobulated, with a low or intermediate signal intensity on $\mathrm{T} 1$ weighted images and a high signal intensity on T2-weighted images (13). MRI allows precise staging of medullar involvement and the soft-tissue mass and helps in diffusion assessment. High-grade tumors do not have septations and show diffuse, heterogeneous enhancement. Low-grade lesions show a lobulated pattern with enhanced septations after intravenous injection of contrast medium but cannot be easily differentiated from benign chondroma (14). Even if size, radiologic aspect, and location are known, biopsy is mandatory $(15,16)$. ${ }^{18} \mathrm{~F}-\mathrm{FDG}$ PET presents with limitations for imaging chondrosarcoma with low cellularity and low vascularity. The treatment is radical surgery. These lesions are not sensitive to the chemotherapy used in dedifferentiated, mesenchymal, or metastatic forms. Radiotherapy is an alternative to incomplete surgery. The staging must be accurate to delineate the limits of the tumor. Follow-up is usually performed by conventional radiography and MRI, even if metallic prostheses that may generate artifacts are present.

In this issue of The Journal of Nuclear Medicine, Miot-Noirault et al. (17) demonstrate that ${ }^{99 \mathrm{~m}} \mathrm{Tc}-\mathrm{NTP} 15-5$ cartilage scintigraphy allows visualization of small tumors in a rat model of grade II chondrosarcoma. More interestingly, ${ }^{99 \mathrm{~m} T c-N T P} 15-5$ cartilage scintigraphy can visualize disease recurrence early after surgery, and that advantage could be the major interest in this setting since morphologic signs shown by CT or MRI are difficult to interpret after surgery. It is not certain whether ${ }^{99 m}$ Tc-NTP $15-5$ cartilage scintigraphy could help with differential diagnosis between benign and malignant tumors since both are proteoglycan-rich, but in cases of sufficient sensitivity, this imaging technique would probably be helpful for grading, follow-up, and detection of recurrence.

\section{OTHER APPLICATIONS}

Besides cartilage imaging, NTP 15-5 may be useful in some cardiovascular diseases involving arterial remodeling, particularly ascending aortic aneurysms and dissections. In these, the alterations of the arterial wall include proteoglycan-rich extensive mucoid degeneration within the medial layer, colocalized with areas of cell disappearance and disruption of extracellular matrix elastic and collagen fibers (18). Another group of investigators found that thrombin, which has an affinity for sulfated glycosaminoglycans, could be retained within these areas. Thus, one can assume that NTP 15-5 would allow the detection and quantification of mucoid degeneration in ascending aortic aneurysms and provide an in vivo imaging marker of arterial wall degeneration.

\section{CONCLUSION}

Indeed, besides anatomic imaging as performed by MRI, there is a real need for functional imaging of cartilage, and proteoglycans represent a good target for it. Even if the uptake mechanism and specificity of ${ }^{99 m}$ Tc-NTP $15-5$ are not completely clear, it represents a valid possibility for this purpose. The major challenge will be obtaining sufficient scintigraphic sensitivity to visualize preclinical disease and to accurately quantify tracer uptake, representing proteoglycan content, for therapeutic evaluation in a preclinical setting of arthrosis. A PET tracer might be more suitable for such quantification.

\section{Laure Sarda-Mantel and Dominique Le Guludec \\ U 773 Inserm et Université Denis Diderot and Service de Médecine Nucléaire, Hôpital Bichat, AP-HP Paris, France}

\section{REFERENCES}

1. Garrigue H, Maurizis JC, Nicolas C, et al. Disposition and metabolism of two acetylcholinesterase reactivators, pyridoxime and HI6, in rats submitted to organophosphate poisoning. Xenobiotica. 1990;20:699-709.

2. Maurizis JC, Ollier M, Nicolas C, Madelmont JC, Garrigue H, Veyre A. In vitro binding of oxime acetylcholinesterase reactivators to proteoglycans synthesized by cultured chondrocytes and fibroblasts. Biochem Pharmacol. 1992;44:1927-1933.

3. Olsen GD, Chan EM, Riker WK. Binding of d-tubocurarine di (methyl- ${ }^{14} \mathrm{C}$ ) ether iodide and other amines to cartilage, chondroitin sulfate and human plasma proteins. J Pharmacol Exp Ther. 1975;195:242-250.

4. Larsson B, Nilsson M, Tjälve H. The binding of inorganic and organic cations and $\mathrm{H}+$ to cartilage in vitro. Biochem Pharmacol. 1981;30:2963-2970.

5. Nicolas C, Verny M, Giraud I, et al. New quaternary ammonium oxicam derivatives targeted toward cartilage: synthesis, pharmacokinetic studies, and antiinflammatory potency. J Med Chem. 1999;42:5235-5240.

6. Maurizis JC, Rapp M, Nicolas C, Ollier M, Verny M, Madelmont JC. Disposition in rats of N-pyridinium-propyl-cyclam, N-triethylammoniumpropyl-cyclam, and N-[triethylammonium]-3-propyl[15] ane-N5, potential cartilage imaging agents. Drug Metab Dispos. 2000;28:418-422.

7. Ollier M, Maurizis JC, Nicolas C, et al. Joint scintigraphy in rabbits with ${ }^{99 \mathrm{~m}} \mathrm{Tc}-\mathrm{N}$-[3-(tiethylammonio)propyl]-15ane-N5, a new radiodiagnostic agent for articular cartilage imaging. $J$ Nucl Med. 2001;42:141-145.

8. Raynauld JP, Martel-Pelletier J, Abram F, et al. Analysis of the precision and sensitivity to change of different approaches to assess cartilage loss by quantitative MRI in a longitudinal multicentre clinical trial in patients with knee osteoarthritis. Arthritis Res Ther. 2008;10:R129.

9. Wang Q, Zheng YP, Qin L, et al. Real-time ultrasonic assessment of progressive proteoglycan depletion in articular cartilage. Ultrasound Med Biol. 2008;34:1085-1092.

10 Pinker K, Szomolanyi P, Welsch GC, et al. Longitudinal evaluation of cartilage composition of matrix-associated autologous chondrocyte transplants with 3-T delayed gadolinium-enhanced MRI of cartilage AJR. 2008;191:1391-1396. 
11. Bastiaansen-Jenniskens YM, Koevoet W, Feijt C, et al. Proteoglycan production is required in initial stages of new cartilage matrix formation but inhibits integrative cartilage repair. J Tissue Eng Regen Med. 2009;3:117-123.

12. Krishnan K. Dahlin's Bone Tumors: General Aspects and Data on 11,087 Cases. 5th ed. Philadelphia, PA: Lippincott-Raven Publishers; 1996.

13. Varma DG, Ayala AG, Carrasco CH, Guo SQ, Kumar R, Edeiken J. Chondrosarcoma: MR imaging with pathologic correlation. Radiographics. 1992;12:687-704.
14. Welkerling H, Kratz S, Ewerbeck V, Delling G. A reproducible and simple grading system for classical chondrosarcomas: analysis of 35 chondrosarcomas and 16 enchondromas with emphasis on recurrence rate and radiological and clinical data. Virchows Arch. 2003;443:725-733.

15. Geirnaerdt MJ, Hermans J, Bloem JL, et al. Usefulness of radiography in differentiating enchondroma from central grade I chondrosarcoma. AJR. 1997;169:1097-1104.

16. Murphey MD, Flemming DJ, Boyea SR, Bojescul JA, Sweet DE, Temple HT. Enchondroma versus chondrosarcoma in the appendicular skeleton: differentiating features. Radiographics. 1998;18: 1213-1237.

17. Miot-Noirault E, Gouin F, Vidal A, et al. First preclinical imaging of primary cartilage neoplasm and its local recurrence using ${ }^{99 \mathrm{~m}} \mathrm{Tc}$ NTP 15-5 radiotracer. J Nucl Med. 2009;50: 1541-1547.

18. Borges LF, Touat Z, Leclercq A, et al. Tissue diffusion and retention of metalloproteinases in ascending aortic aneurysms and dissections. Hum Pathol. 2009;40:306-313

\section{Errata}

The article "Relationship Between Cancer Type and Impact of PET and PET/CT on Intended Management: Findings of the National Oncologic PET Registry," by Hillner et al. ( $\mathrm{Nucl}$ Med. 2008;49:1928-1935), contained an error in the reporting of a previous study. The first paragraph of the "Outcomes" sections should end with the following corrected sentence: "In our previous report (5), we noted that PET led to a change in intended management in 36.5\% of cases; a switch from nontreatment to treatment was 3-fold more likely than the converse." The authors regret the error.

In the article "Initial Evaluation of ${ }^{11} \mathrm{C}-\mathrm{DPA}-713$, a Novel TSPO PET Ligand, in Humans," by Endres et al. ( $\mathrm{N}$ Nucl Med. 2009;50:1276-1282), one source of funding was inadvertently omitted from the Acknowledgments. The corrected funding line is as follows: "This study is supported by a pilot award from The JHU NIMH Center for Novel Therapeutics (to C.J.E.), a grant from The Dana Foundation (to M.G.P.), and the NIMH Toxicological Evaluation of Novel Ligands Program." The authors regret the error. 